\title{
NONLINEAR PREDICTION OF HUMAN RESTING-STATE FUNCTIONAL CONNECTIVITY BASED ON NETWORK COMMUNICATION MEASURES
}

\author{
Fuliang Wang ${ }^{1}$, Xue Chen', Yanjiang Wang ${ }^{3}$ \\ ${ }^{1,2,3}$ College of Information and Control Engineering, China University of Petroleum (East China), Qingdao, P. R. \\ China
}

\begin{abstract}
Mounting evidence demonstrated that neuronal activity derived from functional magnetic resonance imaging (fMRI) relates to the underlying anatomical circuitry measured by diffusion tensor/spectrum imaging (DTI/DSI). However, exploring the relationship between functional connectivity (FC) and structural connectivity (SC) remains challengeable and thus has motivated a number of computational models to investigate the extent to which the dynamics depend on the topology. Nevertheless, most of the models are complex and difficult to treat analytically. In this paper, for simplicity, we utilize four network communication measures extracted from SC as well as polynomial curves fitting method to predict FC. Our results indicate that all of these measures predict FC via the nonlinear fitting method. Besides, compared with the linear method, the fitting value between predicted FC and empirical FC attains higher after applying nonlinear process on communication measures which may help to shed light on the function-structure relationship.
\end{abstract}

Key Words: brain connectivity; fMRI; DTI/DSI; network communication measure; nonlinear fitting

\section{INTRODUCTION}

In order to fully characterize the human connectome and functionally connected networks, there emerges advances in DSI/DTI, fMRI, and related technologies. Usually, there are two kinds of connectivity related to human brain. The structural connectivity (SC) is derived from DTI/DSI and the functional connectivity (FC) is obtained by measuring the correlation of spontaneous Blood Oxygenation LevelDependent (BOLD) fluctuations [1-6]. It has been demonstrated that human brain dynamics relates to the underlying topology. To this end, a growing number of studies have focused on exploring the relationship between FC and SC by network modeling or neural mass modeling [7-12]. However, how FC relates to SC still remains an open question. There exists a problem that most of the computational models are too complex and difficult to treat analytically.

In our present study, for simplicity, we make use of four communication measures extracted from SC - search information, path transitivity, shortest path length, and the number of shortest path steps - as well as polynomial curves fitting method to predict FC. Notably, it has been demonstrated that all of these four communication measures can predict the strength of functional connectivity among both connected and unconnected node pairs by linear regression [13]. On the basis of this, we carry out an extensive comparison of linear regression method and the nonlinear polynomial fitting method while predicting FC from SC. We conclude that the nonlinear curves fitting method performs better when the order of polynomial is appropriate.

\section{MATERIALS AND METHODS}

\subsection{Database}

Two databases, one low-resolution including 66 regions of interest (ROIs), the other one includes 90 ROIs.

The SC matrix of the 66-ROI dataset is based upon the work of [3]. Each element in the SC matrix represents the density with which two different brain regions are connected. The FC matrix was examined by measuring the corresponding fMRI BOLD signals obtained for each brain area during $20 \mathrm{~min}$. Each value in the FC matrix denotes the functional connection between two node pairs [10].

The 90-ROI dataset is a dataset as described in [14]. The structural and diffusion MR volumes were parcellated into 90 cerebral cortical areas after diffusion tractography processing [15]. Resting-state fMRI (rs-fMRI) was acquired with 180 samples. 10 samples at the beginning were discarded. Before rs-fMRI, the subjects were instructed to think of nothing in particular. Finally, the fiber strengths produced by the streamline tractography algorithm were resampled into a Gaussian distribution. Both FC and SC are averaged across the 8 individual participants.

Note that all self-connections (diagonal elements in the FC matrix) are excluded. The resulting SC matrices and FC matrices in the above two databases are shown in Figure.1. Figure.1A shows SC matrix (left panel) and FC matrix (right panel) from 66-ROI database. Figure.1B shows SC matrix (left panel) and FC matrix (right panel) from 90-ROI database. 


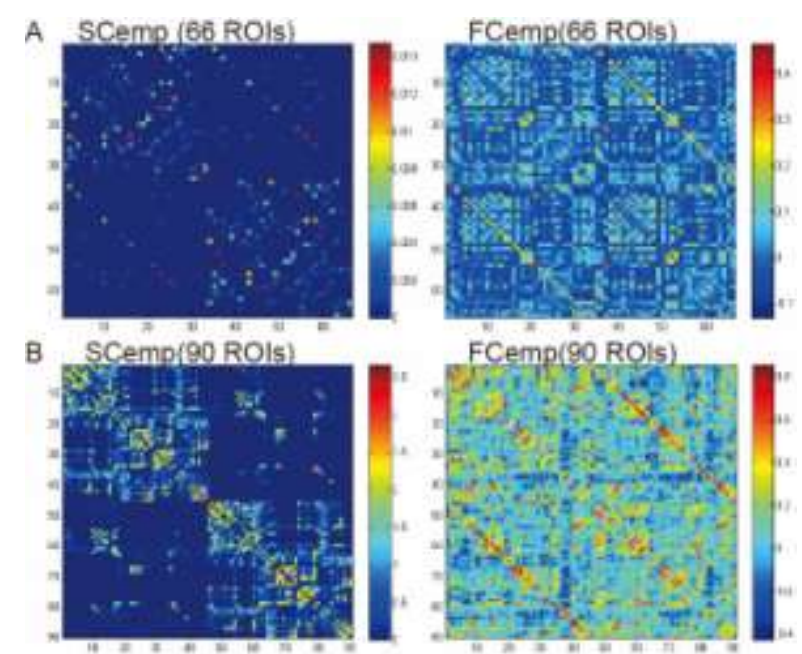

Figure.1. Two database.

\subsection{Communication Measures}

In this paper, we focus on four communication measures: shortest path length, the number of shortest path steps, search information and path transitivity [13].

The SC of a parcellation of the human cortex into $N$ regions can be expressed as undirected weighted graph $G \equiv\{V, W\}$ formed by a set of nodes $V=\left\{v_{1}, v_{2}, \ldots, v_{n}\right\}$ and a matrix of fiber density values $W=\left[w_{i j}\right]$ with values in the range of $[0,1]$, and with $w_{i j}=0$ for regions $i, j$ that are not directly connected. Converting $W$ into a matrix of edge lengths or distances $L=\left[l_{i j}\right]$ (here calculated using the matrix transforms $L=1 / W$ ) allows identification of shortest paths, comprising lists of unique weighted edges, that span the minimum distance between each node pair. A shortest path length ( SPL ) can be described by $\pi_{s \rightarrow t}=\left\{w_{s i}, w_{i j}, \ldots, w_{k t}\right\}$ from a source node $s$ to a target node $t$ and the corresponding nodes can be expressed by $\Omega_{s \rightarrow t}=\{s, i, j, \ldots, k, t\}$. $K$ denotes the number of shortest path steps with $\left|\pi_{s \rightarrow t}\right|=K$, and thus $\left|\Omega_{s \rightarrow t}\right|=K+1$

Search information ( $S$ ) quantifies the accessibility or "hiddenness" of a path linking a source node $s$ to a target node ${ }^{t}$ within the network by measuring the amount of knowledge or information to access the path. Assuming search information travels along the shortest path and given the lack of directionality in the SC matrix, the search information of bidirectional shortest-path $\pi_{s \leftrightarrow t}$ is defined as follows [13]:

$$
\begin{aligned}
& P\left(\pi_{s \rightarrow t}\right)=\prod_{i \in \Omega_{s \rightarrow t}^{*}} \frac{\pi_{i \rightarrow t}^{(1)}}{w_{i}}, \\
& S\left(\pi_{s \rightarrow t}\right)=-\log _{2}\left(P\left(\pi_{s \rightarrow t}\right)\right), \\
& S\left(\pi_{s \leftrightarrow t}\right)=\frac{S\left(\pi_{s \rightarrow t}\right)+S\left(\pi_{t \rightarrow s}\right)}{2}
\end{aligned}
$$

Where $\pi_{i \rightarrow t}^{(1)}$ is the first element (weighted edge) of the path $\pi_{s \leftrightarrow t}$ and $\Omega_{s \rightarrow t}^{*}$ represents the sequence of nodes excluding the target, i.e., $\Omega_{s \rightarrow t}^{*}=\{s, i, j, \ldots, k\} . P\left(\pi_{s \rightarrow t}\right)$ is the probability of taking the shortest path from $s$ to ${ }^{t}$.

Path transitivity ( $P T$ ) describes the density of local detours that are available along the shortest path, i.e.,

$$
\begin{aligned}
& m_{i j}=\frac{\sum_{k \neq i, j}\left(w_{i k}+w_{j k}\right) \Theta\left(w_{i k}\right) \Theta\left(w_{j k}\right)}{\sum_{k \neq j} w_{i k}+\sum_{k \neq i} w_{j k}}, \\
& M_{s \leftrightarrow t}=\frac{2 \sum_{i \in \Omega} \sum_{j \in \Omega} m_{i j}}{|\Omega|(|\Omega|-1)}
\end{aligned}
$$

Where $\Theta\left(w_{i k}\right)=1$ if $w_{i k}>0$ and 0 otherwise. The measure is independent of the directionality of the path and hence ensures $M\left(\pi_{s \rightarrow t}\right)=M\left(\pi_{t \rightarrow s}\right)$.

\section{RESULTS}

\subsection{Prediction of FC Based on Four Single- predictor Models}

In agreement with the previous study [13], all of these four communication measures predict FC. The capacity of every single-predictor was robust based on the shortest paths computed from SC after applying an inverse transform to convert weight to distance (see Table 1 and Table 2). Figure.2A and Figure.3A show the scatter plot of empirical $\mathrm{FC}$ versus the path transitivity and empirical $\mathrm{FC}$ versus the search information based on 66-ROI database and 90-ROI database, respectively, with red dots representing structurally connected pairs, and black dots representing structurally unconnected pairs. The result implies that FC among node pairs including structurally connected pairs and unconnected pairs are higher if the path transitivity is stronger or the search information is weaker.

Ranking the capacity of these four single-predictor models with linear regression method, comprising the shortest path length (SPL), the number of shortest path steps (K), the search information (S) and the path transitivity (PT), the correlation between predicted FC and empirical FC is significant across two databases (see Table 1 and Table 2). The nonlinear polynomial fitting predictions with the path transitivity and the search information are shown in 
Figure.2B and Figure.3B. The fitting between FC and empirical $\mathrm{FC}$ rises rapidly and then retains stable after a critical point across a range of polynomial orders. The curves shown on these scatter diagrams of Figure.2A and Figure.3A indicate the nonlinear fit (blue) at an appropriate polynomial order and linear fit (yellow). More details are shown in Figure.2 and Figure.3.
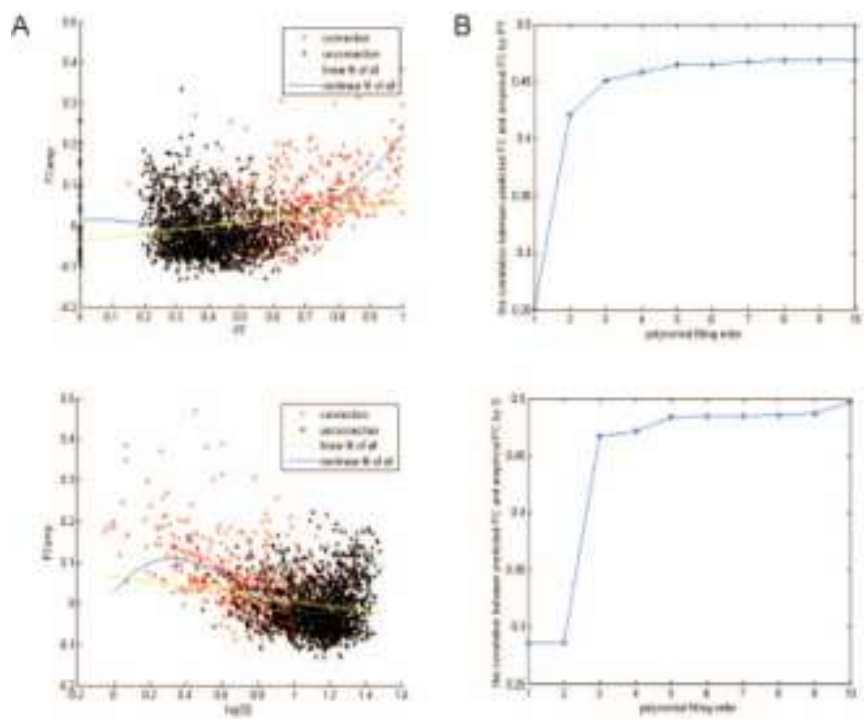

Figure.2. Prediction of FC with path transitivity and search information on 66-ROI database. (A) The scatter plot of empirical FC versus the path transitivity and empirical FC versus the search information with red dots representing structurally connected pairs, and black dots representing structurally unconnected pairs. The yellow line indicates the linear fit, and the blue curve indicates the nonlinear fit at the third polynomial order. (B) The correlation between the empirical FC and predicted FC by the path transitivity and the search information varying with polynomial order.
A
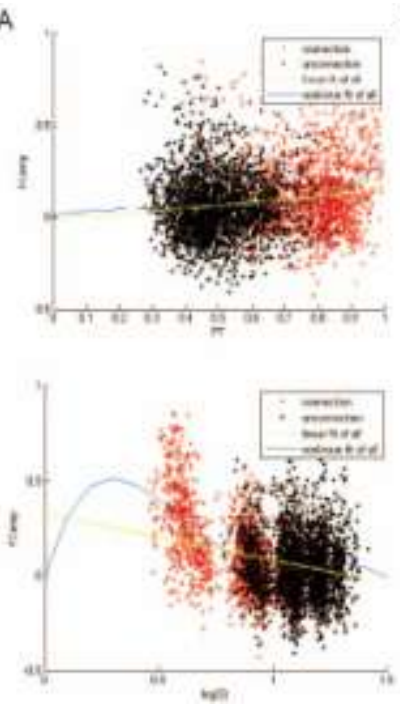
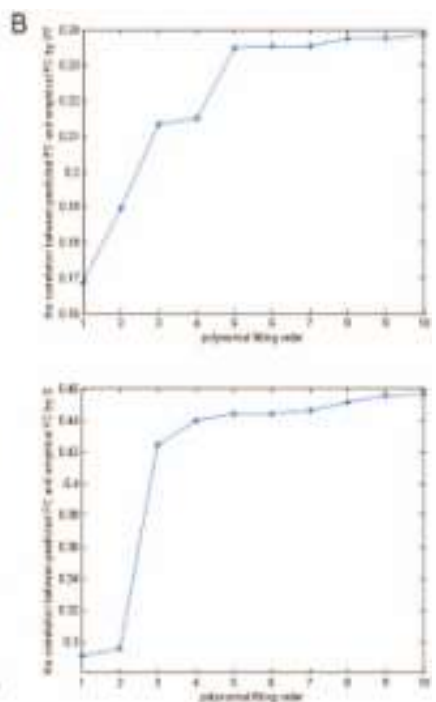

Figure.3. Prediction of FC with the path transitivity and the search information on 90-ROI database. (A) The scatter plot of empirical FC versus the path transitivity and empirical FC versus the search information with red dots representing structurally connected pairs, and black dots representing structurally unconnected pairs. The yellow line indicates the linear fit, and the blue curve indicates the nonlinear fit at the fourth polynomial order. (B) The correlation between the empirical FC and predicted FC by the path transitivity and the search information varying with polynomial order.

Table 1 and Table 2 shows the correlation values between the empirical FC and predicted FC using four singlepredictor models with linear method and nonlinear method at an appropriate polynomial order computed for all pairs $\left(R_{\text {all }}\right)$, only structurally connected pairs $\left(R_{\text {con }}\right)$, and only structurally unconnected pairs $\left(R_{\text {uncon }}\right)$. Assume both search information and path transitivity travel along the shortest path. All correlation values computed for all pairs $\left(R_{\text {all }}\right)$, only structurally connected pairs $\left(R_{c o n}\right)$, and only structurally unconnected pairs $\left(R_{\text {uncon }}\right)$, were significant $(P<0.001)$. SPL denotes shortest path length; $\mathrm{K}$ represents the number of shortest path steps; $\mathrm{S}$ is search information; $\mathrm{PT}$ is path transitivity.

Comparing the effectiveness of prediction with two methods, we can find that every single-predictor model with nonlinear polynomial fitting method shows better performance than with linear regression method among both-hemisphere $(\mathrm{BH})$ prediction, right-hemisphere $(\mathrm{RH})$ prediction and inter-hemisphere $(\mathrm{IH})$ prediction.

\subsection{Prediction of FC Based on Multi-predictor}

\section{Models}

A joint linear multi-predictor model comprising all four communication measures above presents higher capacity of predicting FC than each linear single-predictor model in line with the previous research [13] across two databases (Table 1 and Table 2). Similarly, our results indicate that the joint nonlinear multi-predictor model shows better prediction than each nonlinear single-predictor model and even better performance than the joint linear multi-predictor model. Figrue. 4 shows the patterns of predicted FC simulated by linear multi-predictor model and nonlinear multi-predictor model at a proper polynomial order. Figure.4A demonstrates empirical FC from 66-ROI database (left), the predicted FC simulated by linear multi-predictor model (middle) and the predicted FC simulated by nonlinear multi-predictor model at the seventh polynomial order (right). Figure.4B illustrates empirical FC from 90-ROI database (left), the predicted FC simulated by linear multi-predictor model (middle) and the predicted FC simulated by nonlinear multi-predictor model at the fourth polynomial order (right).

The corresponding correlations between simulated FC and empirical FC of two databases are $R_{\text {all }}=0.4511$ (linear regression model, 66-ROI database), $R_{\text {all }}=0.5283$ (nonlinear regression model, 66-ROI database), $R_{\text {all }}=$ 0.4296 (linear regression model, 90-ROI database) and $R_{\text {all }}=$ 0.4674 (nonlinear regression model, 90-ROI database). 

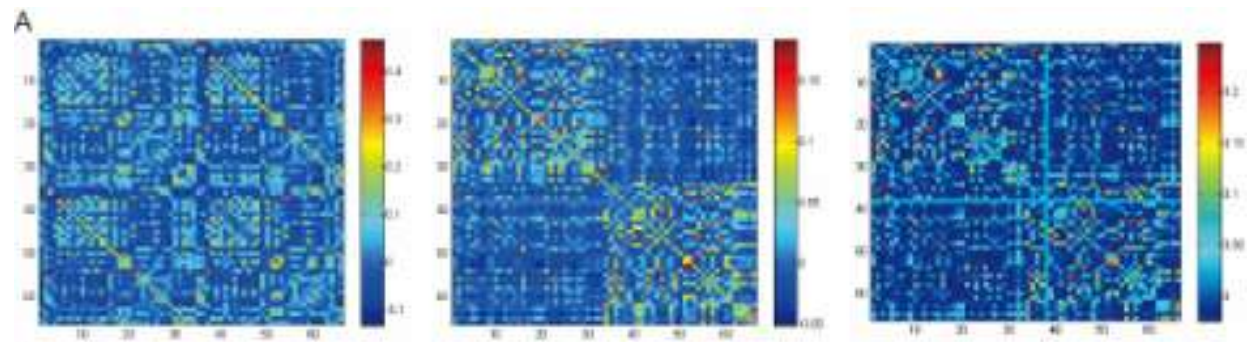

B
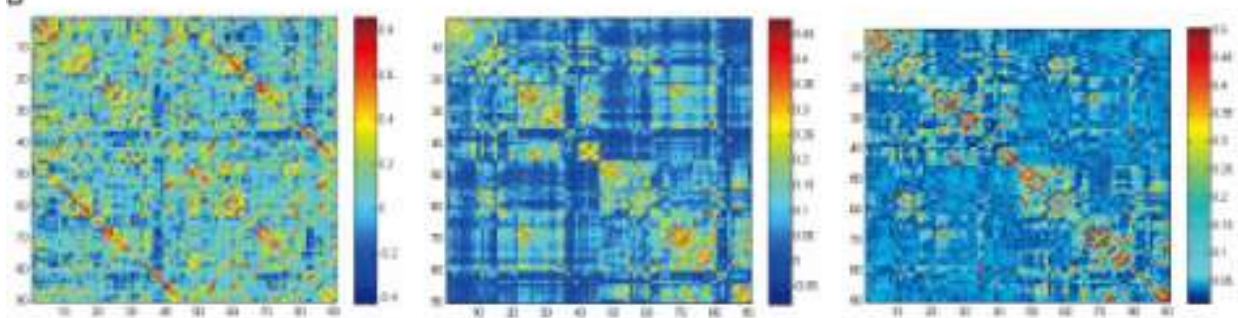

Figure.4. Empirical FC and predicted FC derived from linear and nonlinear regression model across two databases.

Table 1. The prediction of FC based on 66-ROI database using linear single-predictor and multi-predictor models as well as nonlinear single-predictor and multi-predictor models.

\begin{tabular}{|c|c|c|c|c|c|c|c|c|}
\hline \multirow{2}{*}{\multicolumn{2}{|c|}{ FC predictors }} & & \multicolumn{3}{|l|}{ Linear } & \multicolumn{3}{|c|}{ Nonlinear } \\
\hline & & & BH & RH & IH & $\mathbf{B H}$ & $\mathbf{R H}$ & IH \\
\hline \multirow{4}{*}{ SPL } & \multirow{3}{*}{$\log (\mathrm{SPL})$} & $R_{\text {all }}$ & 0.1476 & 0.3163 & 0.1043 & 0.4285 & 0.5048 & 0.4255 \\
\hline & & $R_{\text {con }}$ & 0.5011 & 0.5081 & 0.5018 & 0.5545 & 0.6279 & 0.5160 \\
\hline & & $R_{\text {uncon }}$ & 0.0193 & -0.0056 & 0.0066 & 0.1139 & 0.1203 & 0.2057 \\
\hline & & $R_{\text {all }}$ & 0.3094 & 0.3908 & 0.2266 & 0.4276 & 0.5408 & 0.3544 \\
\hline \multirow[t]{3}{*}{ K } & \multirow[t]{3}{*}{$\log (\mathrm{K})$} & $R_{\text {con }}$ & 0.4922 & 0.5008 & 0.5295 & 0.5261 & 0.5618 & 0.5303 \\
\hline & & $R_{\text {uncon }}$ & 0.0073 & -0.0454 & -0.0005 & 0.2172 & 0.2679 & 0.2380 \\
\hline & & $R_{\text {all }}$ & 0.2854 & 0.3944 & 0.2034 & 0.4669 & 0.5292 & 0.4341 \\
\hline \multirow[t]{3}{*}{ S } & \multirow[t]{3}{*}{$\log (\mathrm{S})$} & $R_{\text {con }}$ & 0.5357 & 0.5914 & 0.6146 & 0.4873 & 0.4952 & 0.6217 \\
\hline & & $R_{\text {uncon }}$ & 0.0347 & 0.0324 & 0.0140 & 0.2572 & 0.3220 & 0.2640 \\
\hline & & $R_{\text {all }}$ & 0.2504 & 0.2873 & 0.1917 & 0.4506 & 0.4455 & 0.4865 \\
\hline \multirow[t]{2}{*}{ PT } & \multirow[t]{2}{*}{ PT } & $R_{\text {con }}$ & 0.3996 & 0.3051 & 0.5138 & 0.5151 & 0.4546 & 0.6201 \\
\hline & & $R_{\text {uncon }}$ & -0.1498 & -0.1572 & -0.1687 & 0.1683 & 0.0884 & 0.2322 \\
\hline \multirow{3}{*}{\multicolumn{2}{|c|}{$\begin{array}{l}\text { All measure } \\
\text { predictors }\end{array}$}} & $R_{\text {all }}$ & 0.4511 & 0.5502 & 0.3951 & 0.5283 & 0.5890 & 0.5067 \\
\hline & & $R_{\text {con }}$ & 0.5442 & 0.5930 & 0.6401 & 0.6076 & 0.6320 & 0.6285 \\
\hline & & $R_{\text {uncon }}$ & 0.1143 & 0.1989 & 0.0911 & 0.2758 & 0.2795 & 0.3147 \\
\hline
\end{tabular}

Table 2. The prediction of FC based on 90-ROI database using linear single-predictor and multi-predictor models as well as nonlinear single-predictor and multi-predictor models.

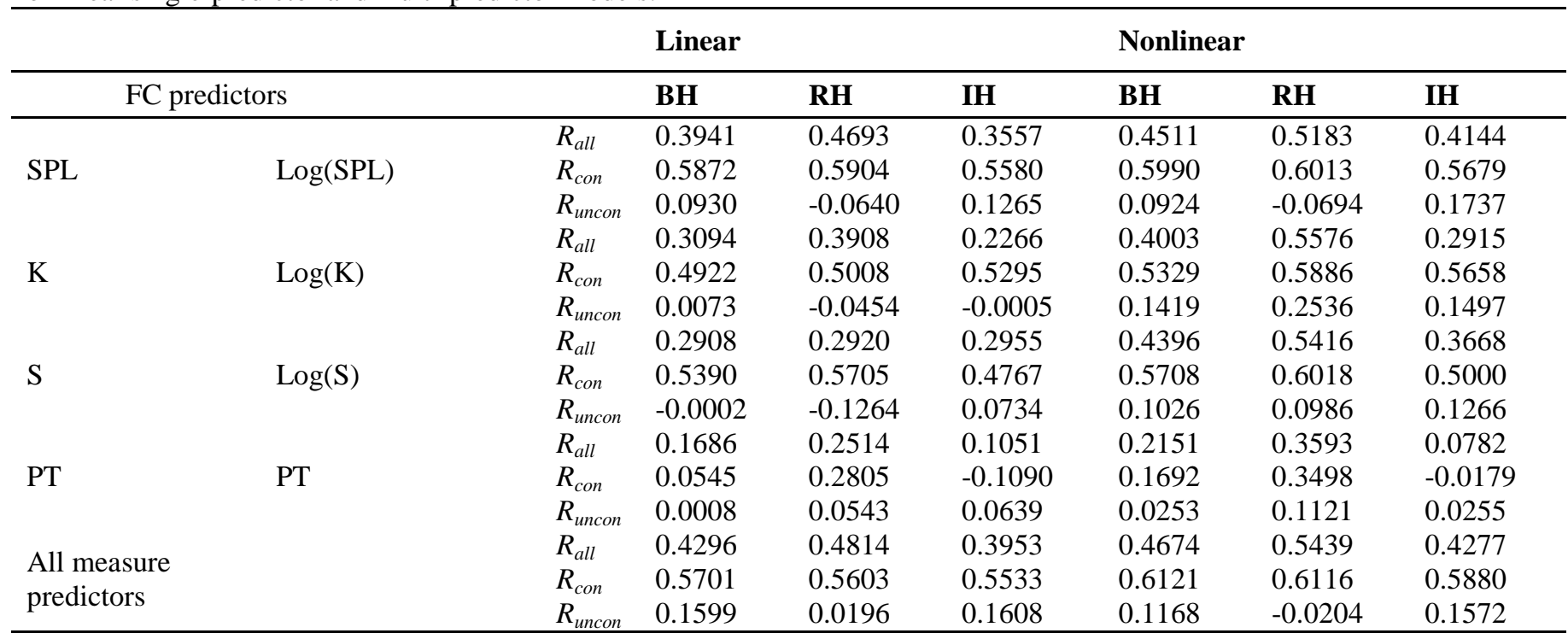




\section{DISCUSSION}

Since the mid 1990s, the dynamics of resting-state brain triggered much interest in the study of neuroscience. Several large-scale computational models were proposed to explore the relationship between the anatomical structure and the functional dynamics. Despite the difference between models, the model parameters were adjusted when the system operates at the critical point (i.e. the fitting between the empirical FC and simulated FC is optimal when the system operates at the edge of an instability) [16]. For simplicity, here four communication measures are extracted from SC in order to predict FC without looking for the instable point.

We have demonstrated that each communication measure shows better capacity of predicting FC by means of nonlinear polynomial fitting model than linear regression model. A joint nonlinear multi-predictor model presents stronger prediction across two databases among structurally connected pairs, structurally unconnected pairs and all node pairs. Nevertheless, the capacity of predicting interhemispheric links is weak relatively which may restrict the simulation of all node pairs accordingly. Making a thorough research on inter-hemispheric connections seems to be vital. Besides, we find there exists few structurally isolated vertices while show functional correlations with other vertices. The inaccuracy of DTI/DSI might lead to the existence of these points. Evidence has documented that there is an inherent limitation in determining long-range anatomical projections by diffusion MRI tractography [17]. One of the great significances of the research on the structure-function relation is offering help for health and disease. Resting-state alterations have been found in Alzheimer's disease (AD), schizophrenia, dementia and many other mental diseases which attract a mounting number of studies in functional and anatomical brain networks [18-20]. Our research probably help understand these challenges and questions in human disease.

\section{ACKNOWLEDGEMENT}

This work was supported by the China Scholarship Council (201306455001) and the National Natural Science Foundation of P.R. China (Grant No. 61271407). We would like to thank Olaf Sporns and Farras Abdelnour for sharing the 66-ROI and 90-ROI SC and FC datasets, respectively.

\section{REFERENCES}

[1]. Greicius MD, Krasnow B, Reiss AL, Menon V, 2003. Functional connectivity in the resting brain: a network analysis of the default mode hypothesis. Proceedings of the National Academy of Sciences of the United States of America 100(1):253-258.

[2]. Fox MD, Raichle ME, 2007. Spontaneous fluctuations in brain activity observed with functional magnetic resonance imaging. Nature Reviews Neuroscience 8(9):700711.

[3]. Hagmann P, Cammoun L, Gigandet X, Meuli R, et al., 2008. Mapping the structural core of human cerebral cortex. PLoS biology 6(7):e159.
[4]. Hagmann P, Cammoun L, Gigandet X, Gerhard S, et al., 2010. MR connectomics: Principles and challenges. Journal of neuroscience methods 194(1):34-45.

[5]. Sporns O, 2011. The human connectome: a complex network. Annals of the New York Academy of Sciences 1224:109-125.

[6]. Tristan T. Nakagawa, Viktor K. Jirsa, Andreas Spiegler, Anthony R. McIntosh, et al., 2013. Bottom up modeling of the connectome: Linking structure and function in the resting brain and their changes in aging. NeuroImage 80:318-329.

[7]. Honey CJ, Kotter R, Breakspear M, Sporns O, 2007. Network structure of cerebral cortex shapes functional connectivity on multiple time scales. Proceedings of the National Academy of Sciences of the United States of America 104(24):10240-10245.

[8]. Ghosh A, Rho Y, McIntosh AR, Kotter R, et al., 2008. Noise during Rest Enables the Exploration of the Brain's Dynamic Repertoire. Plos Computational Biology 4(10).

[9]. Deco G, Jirsa V, McIntosh AR, Sporns O, et al., 2009. Key role of coupling, delay, and noise in resting brain fluctuations. Proceedings of the National Academy of Sciences of the United States of America 106(25):1030210307.

[10]. Honey CJ, Sporns O, Cammoun L, Gigandet X, et al., 2009. Predicting human resting-state functional connectivity from structural connectivity. Proceedings of the National Academy of Sciences of the United States of America 106(6):2035-2040.

[11]. Deco G, Jirsa VK, McIntosh AR, 2011. Emerging concepts for the dynamical organization of resting-state activity in the brain. Nature Reviews Neuroscience 12(1):43-56.

[12]. Deco G, Ponce-Alvarez A, Mantini D, Romani GL, et al., 2013. Resting-State Functional Connectivity Emerges from Structurally and Dynamically Shaped Slow Linear Fluctuations. Journal of Neuroscience 33(27):11239-11252.

[13]. Goni J, van den Heuvel MP, Avena-Koenigsberger A, de Mendizabal NV, et al., 2014. Resting-brain functional connectivity predicted by analytic measures of network communication. Proceedings of the National Academy of Sciences of the United States of America 111(2):833-838.

[14]. Abdelnour F, Voss HU, Raj A, 2014. Network diffusion accurately models the relationship between structural and functional brain connectivity networks. NeuroImage 90:335-347.

[15]. Tzourio-Mazoyer N, Landeau B, Papathanassiou D, Crivello F, et al., 2002. Automated anatomical labeling of activations in SPM using a macroscopic anatomical parcellation of the MNI MRI single-subject brain. NeuroImage 15(1):273-289.

[16]. Cabral J, Kringelbach ML, Deco G, 2014. Exploring the network dynamics underlying brain activity during rest. Progress in neurobiology 114:102-131.

[17]. Thomas C, Ye FQ, Irfanoglu MO, Modi P, et al., 2014. Anatomical accuracy of brain connections derived from diffusion MRI tractography is inherently limited. Proceedings of the National Academy of Sciences of the United States of America 111(46):16574-16579. 
[18]. Rombouts SARB, Damoiseaux JS, Goekoop R, Barkhof F, et al., 2009. Model-Free Group Analysis Shows Altered BOLD FMRI Networks in Dementia. Human Brain Mapping 30(1):256-266.

[19]. Bassett DS, Nelson BG, Mueller BA, Camchong J, et al., 2012. Altered resting state complexity in schizophrenia. NeuroImage 59(3):2196-2207.

[20]. Binnewijzend MAA, Schoonheim MM, Sanz-Arigita E, Wink AM, et al., 2012. Resting-state fMRI changes in Alzheimer's disease and mild cognitive impairment. Neurobiology of Aging 33(9):2018-2028.

\section{BIOGRAPHIES}

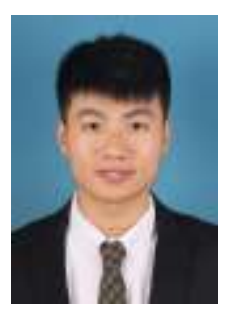

Fuliang Wang received the bachelor's degree from China University of Petroleum (East China) in 2013. He is currently a postgraduate with College of Information and Communication Engineering, China University of Petroleum (East China). His research interests include neural networks and pattern recognition.

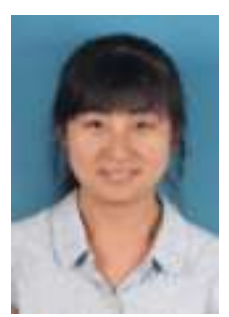

Chen Xue received the bachelor's degree from China University of Petroleum (East China) in 2013. She is currently a Ph.D. student with College of Control theory and Control Engineering, China University of Petroleum (East China). Her research interests include neural networks and neural mass model, neuroscience.

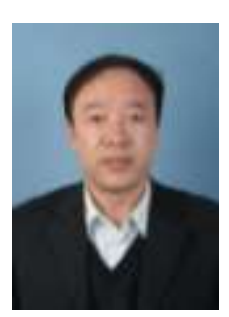

Yanjiang Wang received the M.S. degree from Beijing University of Aeronautics and Astronautics, Beijing, China, in 1989 and the Ph.D. degree from Beijing Jiaotong University, Beijing, China, in 2001. Now he is a professor of the College of Information and Control Engineering, China University of Petroleum, Qingdao, China. He is also the head of the Institute of Signal and Information Processing, China University of Petroleum. Currently, his research interests include bio-inspired pattern recognition, cognitive memory modeling, and human brain connectivity. 